\title{
Early antithrombotic therapy after aortic valve replacement with tissue valves: When the practice diverges from the guidelines
}

\author{
Francesca di Marco, MD, ${ }^{a}$ Massimo Giordan, MD, and Gino Gerosa, MD ${ }^{\mathrm{b}}$
}

See related letter to the editor on page 1425 .

Supplemental material is available online.

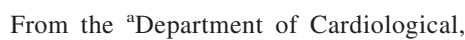
Thoracic, and Vascular Sciences, Division of Cardiac Surgery, Padua University Medical School, Padova, Italy; 'Interventional Cardiology-Cardiology Division, Ospedale Civile Rovigo, Rovigo, Italy.

Please note that the full reference list is available in the online version.

Received for publication Dec 20, 2005; accepted for publication Jan 24, 2006.

Address for reprints: Francesca di Marco, MD, Via Giustiniani 2, 35100 Padova, Italy (E-mail: maxgiordan@yahoo.it).

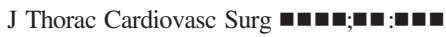

0022-5223/\$32.00

Copyright (C) 2006 by The American Association for Thoracic Surgery

doi:10.1016/j.jtcvs.2006.01.045
$\mathrm{T}$ The American College of Cardiology (ACC) and American Heart Association (AHA) practice guidelines (dating back to 1998) propose early anticoagulation with warfarin as a class I recommendation in patients with biologic prosthetic heart valves, with a target international normalized ratio (INR) of 2.5 to 3.5 , during the first 3 months from the implant. The guidelines do not make any distinction among type of prosthesis (biologic or mechanical) or site of implant. ${ }^{\text {E1 }}$

The first 3 postoperative months are classically considered a high-risk time interval for thromboemboli formation, as long as the process of endothelialization of sewing ring and sutures reaches completion. ${ }^{\mathrm{E} 2}$ Biologic valves have been recognized to be at lower risk when compared with mechanical valves ${ }^{\mathrm{E} 3}$; among them, aortic bioprostheses show inferior thrombogenicity than mitral bioprostheses. ${ }^{\text {E4 }}$

Last year, the CTSNet Valve Technology Center released the results of its study, ${ }^{\mathrm{E} 5}$ which focused on the early antithrombotic regimen adopted by CTSNet Surgeons members, after aortic tissue valve replacement. The study intended to establish the level of awareness of the ACC/AHA guidelines among the 726 participating surgeons and the adherence to the guidelines in their daily practice. In each country (mainly the United States and Europe), the percentage of respondents acquainted with or unaware of the guidelines were equally distributed, with an overall prevalence of awareness (79\% vs $21 \%$ ).

Three main issues emerged from the study:

1. More than $60 \%$ of surgeons are convinced that oral anticoagulation therapy administration prolongs hospital stay of approximately 2 to 3 days.

2. More than $60 \%$ believe that antiplatelet therapy alone represents a valuable alternative, in absence of comorbidities, granting patients safety and reducing overall stay and cost of care; they no longer consider warfarin to be the gold standard of early antithrombotic therapy for biologic valves.

3. Approximately $50 \%$ of surgeons adopt antiplatelet therapy in their current practice instead of warfarin in $90 \%$ of patients without comorbidities.

At the opposite end of the spectrum, more than $25 \%$ of surgeons not only administer warfarin after aortic tissue valve replacement but also maintain long-term anticoagulant therapy, even in the absence of comorbidities.

The emerging issue is that surgeons are not comfortable with the current recommendations and are experimenting with experiencing alternative options. Participating surgeons showed a weak compliance with early anticoagulation therapy prescribed by guidelines, which is mainly related to the intrinsic limits of dicumarolic therapy, such as the need for strict INR monitoring, with progressive dosage adjustment until anticoagulation optimization is achieved, as well as the constant risk of bleeding. ${ }^{\text {E6 }}$ Antiaggregant therapy with aspirin represents an easy alternative and has been adopted by more than $50 \%$ of surgeons, with an actual constant trend diverging from the recommendations. ${ }^{\text {E7-8 }}$ Nevertheless, evidence-based medicine requires proven results before modifying an established practice and adopting new therapeutic strategies.

In an article entitled "Aspirin or Coumadin as the Drug of Choice for Valve Replacement with Porcine Bio-prosthesis," Nunez and colleagues ${ }^{\mathrm{E} 9}$ launched a Hamlet-like dilemma. The study was limited by its retrospective character and 
heterogeneous patient population (patients undergoing aortic, mitral, or double-valve surgery, either in sinus rhythm or atrial fibrillation). Nevertheless, the results outline the absence of thromboembolic complications in patients in sinus rhythm, regardless of whether they received an antiplatelet or anticoagulation regimen. On the other hand, a statistically significant higher prevalence of hemorrhagic complications occurred in patients receiving warfarin.

Ten years later, in a retrospective review, Blair et $\mathrm{al}^{\mathrm{E} 10}$ reported a similar rate of thromboembolic complications after valve replacement with aortic pericardial bioprostheses in patients receiving aspirin $(0.8 \% \pm 0.2 \%$ patients/year $)$, warfarin $(2.9 \% \pm 1.6 \%$ patients/year), or no therapy $(1.5 \%$ $\pm 0.6 \%$ patients/year).

In another retrospective trial, Moinuddeen and colleagues ${ }^{\mathrm{E} 11}$ claimed that anticoagulation therapy after biologic aortic valve replacement is unnecessary. In fact, even if no increase in postoperative rate bleeding or in prolonged hospitalization was registered in anticoagulated patients, survival was not affected by the exclusion of any antithrombotic therapy.

This article was published in the following issue of the same journal reporting the ACC/AHA practice guidelines, representing a clear attempt of questioning the guidelines and the existence of different policies in clinical practice. Ticlopidine therapy has also been proposed ${ }^{\mathrm{E} 12}$ as an effective alternative for thromboembolism prevention (linearized incidence of $0.5 \%$ patients/year vs $3 \%$ patients/year in those receiving Coumadin, $P<.001$ ), unfortunately encumbered by a high rate of hemorrhagic events $(0.75 \%$ patients/year).

As Vaughan et al remarks, ${ }^{\mathrm{E} 13}$ in the consensus report of 2001, the position of the American College of Chest Physicians regarding antithrombotic early management of bioprostheses in aortic position was less strict compared with the position of the AHA/ACC, indicating 3 months of postoperative anticoagulation as a Grade $2 \mathrm{C}$ recommendation. Nevertheless, at the same time, a goal INR of 2.5 (and we wonder how this can be reached without vitamin K antagonist therapy) during the first 3 months was suggested for both aortic and mitral valves by the American College of Chest Physicians as a Grade A1 recommendation. ${ }^{\text {E14,E15 }}$ More recently, the 7th ACPP conference recommended either vitamin $\mathrm{K}$ antagonists (Grade 2C) or aspirin (Grade 1C) as antithrombotic prophylaxis during the first 3 months after aortic bioprosthetic implant. On the basis of observational trials, the evidence for anticoagulation therapy's requirement for aortic tissue valves is weaker compared with that for mitral valves. Nevertheless, the opportunity to alternatively adopt an antiplatelet therapy is not supported by firm and undisputable evidence. Convincing data now support the adoption of aspirin after aortic valve replacement with biologic valves and may lead to guidelines revisitation. Gherli and associates ${ }^{\mathrm{E} 16}$ conducted a prospective evaluation on 275 consecutive patients in sinus rhythm who received aspirin or warfarin postoperatively according to the surgeon's personal preferences. Warfarin did not confer further advantages in terms of stroke-free and overall survival.

A retrospective, large analysis focusing on postoperative cerebral adverse events after tissue aortic valve surgery confirmed the absence of added benefit from early anticoagulation adoption. ${ }^{\mathrm{E} 17}$ In the discussion of the article, it is pointed out that a large randomized study would be required to exclude the necessity of anticoagulation in the first 3 months after the implant, but it seems that the current practice is advancing faster than the literature. Antiplatelet therapy is imposing as a widely accepted alternative, as a "fait accompli," with an evident tendency to abandon the established guidelines, which might not be as safe for surgeons as for patients. In fact, as admitted by Sundt and colleagues, guidelines are no longer only a reference advice for the daily medical practice, but in case of legal litigation they represent a written law we might be accused to have inconveniently dismissed.

Taking this in consideration, as we have already highlighted, ${ }^{\text {E18 }}$ we believe that mere clinical evaluation of postoperative neurologic complications in patients treated with antiplatelet therapy instead of warfarin may be restrictive. In fact, microembolization phenomena might not be clinically evident, and their occurrence might not be excluded on the basis of neurologic evaluation. Cerebral computed tomography scan would probably provide the most exhaustive information, but cost and patients' radiography exposition limit its wide use as a screening test. Instead, we propose a parallel clinical observation and evaluation of microembolic signals through the transcranial Doppler (TCD), a noninvasive ultrasound examination.

TCD Doppler microembolic signals, called high-intensity transient signals (HITS), were first identified in the late 1990s during coronary surgery performed using extracorporeal circulation. ${ }^{\mathrm{E} 19}$ A causal relation to postoperative cognitive dysfunction with possible deterioration of both working and episodic memory has been hypothesized. ${ }^{\text {E20 }}$

HITS have been detected during the first 6 months after valve surgery with mechanical prostheses, even if different pathophysiologic mechanisms have been claimed to be involved in their genesis. HITS detected in recipients of mechanical valves have been interpreted as the Doppler photograph of cavitation phenomena with gas bubble formation generating at the valve closure. ${ }^{\text {E21 }}$ Neoformed gaseous microbubbles would be responsible for the platelets' activation and subsequent hypercoagulability state, exposing patients to a higher risk of thromboembolic accidents. ${ }^{\text {E22 }}$ Furthermore, concomitant independent formation of thrombotic aggregates cannot be excluded. On the contrary, HITS have rarely been reported in bioprostheses recipients. ${ }^{\mathrm{E} 23, \mathrm{E} 24}$ In this case, a cavitation mechanism can- 
not be addressed ${ }^{\mathrm{E} 25}$; instead, transient solid embolization may offer a reasonable explanation.

At this point, HITS detection may be useful to investigate any possible difference in solid microembolization during the first 3 postoperative months, which are traditionally believed to be the high-risk period for thromboembolic complications. ${ }^{\text {E26 }}$

Because some thromboembolic phenomena may be clinically irrelevant, we believe that a clinical neurologic evaluation associated with TCD screening for HITS detection during the early postoperative period may be a reliable support to a meaningful comparison of the neurologic course in patients undergoing different antithrombotic drug regimens. For this reason, at our institution we evaluated HITS occurrence after valve replacement surgery. In the subcohort of aortic bioprostheses recipients receiving aspirin instead of warfarin, who were free from neurologic complications, no HITS were detected at TCD. Subsequently, we decided to resume the study randomizing patients to anticoagulant or antiplatelet therapies and evaluating HITS occurrence in all, with encouraging preliminary results that will be available soon.

On the basis of our experience, we believe that TCD may be a valuable tool to complete the information gained through deep neurologic clinical examination, further increasing the likelihood of an uneventful postoperative course. We are convinced that the firm instrumentation-based evidence offered by TCD is crucial for an aware patient's antithrombotic management, especially when practice differs from established guidelines.

We must remain aware that guidelines will not change because cardiac surgeons simply choose different ways from those suggested, but because enough evidence is provided to support alternative therapeutic policies.

\section{References}

E1. Bonow RO, Carabello B, de Leon AC, et al. ACC/AHA Guidelines for the management of patients with valvular heart disease: executive summary. A report of the American College of Cardiology/American Heart Association Task Force on Practice Guidelines (Committee on Management of Patients with Valvular Heart Disease). J Heart Valve Dis. 1998;7:672-707.

E2. Becker RC, Eisenberg P, Turpie AGG. Pathobiologic features and prevention of thrombotic complications associated with prosthetic heart valves: fundamental principles and the contribution of platelets and thrombin. Am Heart J. 2001;141:1025-37.

E3. Fradet GJ, Jamieson WR, Abel JG, et al. Thromboembolic and hemorrhagic risk in mechanical and biological aortic prostheses. Ann Thorac Surg. 1995;60(Suppl 2):S453-8.

E4. Turpie AGG, Hirsh J, Gunstensen J, Nelson H, Gent M. Randomised comparison of two intensities of oral anticoagulant therapy after tissue heart valve replacement. The Lancet. 1998;331:1242-45.

E5. http://www.ctsnet.org/file/AnticoagulationSurveyFinalResultsSlidesPDF. pdf.
E6. Agnelli G. Current issues in anticoagulation. Pathophysiol Haemost Thromb. 2005;34(Suppl I):2-9.

E7. http://www.escardio.org//knowledge/ehs/survey/Valvular_Hear_Disease. htm.

E8. Vaughan P, Waterworth PD. An audit of anticoagulation practice among UK cardiothoracic consultant surgeons following valve replacement/repair. J Heart Valve Dis. 2005;14:576-82.

E9. Nunez L, Aguado MG, Celemin D, Iglesias A, Larrea JL. Aspirin or Coumadin as the drug of choice for valve replacement with porcine bioprosthesis. Ann Thorac Surg. 1982;33:354-8.

E10. Blair KL, Hatton AC, White WD, et al. Comparison of anticoagulation regimens after Carpentier-Edwards aortic or mitral valve replacement. Circulation. 1994;90:II214-9.

E11. Moinuddeen K, Quin J, Shaw R, et al. Anticoagulation is unnecessary after biological aortic valve replacement. Circulation. 1998;98: II95-8; discussion II98-9.

E12. Aramendi JL, Agredo J, Llorente A, Larrarte C, Pijoan J. Prevention of thromboembolism with ticlopidine shortly after valve repair or replacement with a bioprosthesis. J Heart Valve Dis. 1998;7:610-4.

E13. Vaughan P, Waterworth PD. Anticoagulation after bioprosthetic aortic valve replacement. J Thorac Cardiovasc Surg. In press.

E14. Stein PD, Alpert JS, Bussey HI, et al. Antithrombotic therapy in patients with mechanical and biological prosthetic heart valves. Chest. 2001;119:(Suppl 1)220S-7S.

E15. Salem DN, Stein PD, Al-Ahmad A, et al. Antithrombotic therapy in valvular heart disease-native and prosthetic: the seventh ACPP conference on antithrombotic and thrombolytic therapy. Chest. 2004; $126: 457-82$.

E16. Gherli T, Colli A, Fragnito C, et al. Comparing warfarin with aspirin after biological aortic valve replacement: a prospective study. Circulation. 2004;110:496-500.

E17. Sundt TM, Zehr KJ, Dearani JA, et al. Is early anticoagulation with warfarin necessary after bioprosthetic aortic valve replacement? J Thorac Cardiovasc Surg. 2005;129:1024-31.

E18. di Marco F, Meneghetti G, Gerosa G. Early anticoagulation after aoartic valve replacement with bioprostheses: time to abandon it? J Thorac Cardiovasc Surg. 2005;130:1482-3.

E19. Barbut D, Yao FS, Hager DN, et al. Comparison of transcranial Doppler ultrasonography and transesophageal echocardiography to monitor emboli during coronary artery bypass surgery. Stroke. 1996; 27:87-90.

E20. Pugsley W, Klinger L, Paschalis C, Treasure T, Harrison M, Newman $\mathrm{S}$. The impact of microemboli during cardiopulmonary bypass on neuropsychological functioning. Stroke. 1994;25:1393-99.

E21. Milo S, Rambod E, Gutfinger C, Gharib M. Mitral mechanical valves: in vitro studies of their closure, vortex and microbubble formation with possible medical implications. Eur J Cardio-thorac Surg. 2003; 24:364-70

E22. Geiser T, Sturzenegger M, Genewein U, et al. Mechanisms of cerebrovascular events as assessed by procoagulant activity, cerebral microemboli, and platelet microparticles in patients with prosthetic heart valves. Stroke. 1998;29:1770-7.

E23. Sliwka U, Georgiadis D. Clinical correlations of Doppler microembolic signals in patients with prosthetic cardiac valves: analysis of 580 cases. Stroke. 1998;29:140-3.

E24. Eicke BM, Barth V, Kukowski B, Werner G, Paulus W. Cardiac microembolism: prevalence and clinical outcome. J Neurol Sci. 1996; 136:143-7

E25. Gorman M. Transcranial Doppler assessment of cerebral embolic disorders. Progress in Cardiovasc Disease. 2000;2:135-50.

E26. Heras M, Chesebro JH, Fuster V, et al. High risk of thromboemboli early after bioprosthetic cardiac valve replacement. JACC. 1995;25: 1111-9. 